\title{
ON NEW STRENGTHENED HARDY-HILBERT'S INEQUALITY
}

\author{
BICHENG YANG \\ Department of Mathematics \\ Guangdong Education College \\ Guangzhou, Guangdong 510303, P.R. CHINA \\ and \\ LOKENATH DEBNATH \\ Department of Mathematics \\ University of Central Florida \\ Orlando, Florida 32816, U.S.A.
}

(Received February 27, 1997 and in revised form September 8, 1997)

ABSTRACT. In this paper, a new inequality for the weight coefficient $\omega(q, n)$ in the form

$$
\omega(q, n):=\sum_{m=1}^{\infty} \frac{1}{m+n}\left(\frac{n}{m}\right)^{1 / q}<\frac{\pi}{\sin (\pi / p)}-\frac{1}{2 n^{1 / p}+n^{-1 / q}}\left(q>1, \frac{1}{p}+\frac{1}{q}=1, n \in N\right)
$$

is proved. This is followed by a strengthened version of the Hardy-Hilbert inequality.

KEY WORDS AND PHRASES: Hardy-Hilbert's inequality, weight coefficient, Holder's inequality. 1991 AMS SUBJECT CLASSIFICATION CODES: 26D15.

\section{INTRODUCTION}

If $a_{n} \geq 0,0<\sum_{n=1}^{\infty} n^{2} a_{n}^{2}<\infty$, then the Karlson's inequality is

$$
\left(\sum_{n=1}^{\infty} a_{n}\right)^{4}<\pi^{2} \sum_{n=1}^{\infty} a_{n}^{2} \sum_{n=1}^{\infty} n^{2} a_{n}^{2}
$$

where the constant $\pi^{2}$ cannot be made smaller. However, it can be strengthened (see Mikhlin [1], p. 7) as

$$
\left(\sum_{n=1}^{\infty} a_{n}\right)^{4}<\pi^{2} \sum_{n=1}^{\infty} a_{n}^{2} \sum_{n=1}^{\infty}\left(n-\frac{1}{2}\right)^{2} a_{n}^{2} .
$$

In recent years, considerable attention has been given to develop some types of strengthened inequality (see [2]-[10]) by estimating the weight coefficient $\omega(q, n)$ as

$$
\omega(q, n)=\sum_{m=1}^{\infty} \frac{1}{(m+n)}\left(\frac{n}{m}\right)^{1 / q}\left(q>1, p^{-1}+q^{-1}=1, n \in N\right) .
$$

Some improvement of Hardy-Hilbert's inequality (see Hardy et al. [11]) has been made in the form

$$
\sum_{m=1}^{\infty} \sum_{n=1}^{\infty} \frac{a_{m} b_{n}}{m+n}<\frac{\pi}{\sin (\pi / p)}\left\{\sum_{n=1}^{\infty} a_{n}^{p}\right\}^{1 / p}\left\{\sum_{n=1}^{\infty} b_{n}^{q}\right\}^{1 / q} .
$$

In their recent work, $\mathrm{Xu}$ and Gau [2] considered the following weight coefficient (1.3) and proved the following inequality 


$$
\omega(q, n)<\frac{\pi}{\sin (\pi / p)}-\frac{\eta_{p}}{n^{1 / p}+n^{-1 / q}}, \quad \eta_{p}=p-1 .
$$

Then a strengthened Hardy-Hilbert's inequality

$\sum_{m=1}^{\infty} \sum_{n=1}^{\infty} \frac{a_{m} b_{n}}{m+n}<\left\{\sum_{n=1}^{\infty}\left[\frac{\pi}{\sin (\pi / p)}-\frac{p-1}{n^{1 / p}+n^{-1 / q}}\right] a_{n}^{p}\right\}^{1 / p}\left\{\sum_{n=1}^{\infty}\left[\frac{\pi}{\sin (\pi / p)}-\frac{q-1}{n^{1 / q}+n^{-1 / p}}\right] b_{n}^{q}\right\}^{1 / q}$.

was proved. The key is to estimate the corresponding weight coefficient effectively. Hsu and Wang [3] proved the following inequality

$$
\omega(2, n)<\pi-\frac{\theta}{\sqrt{n}}, \quad \theta=\frac{3}{\sqrt{2}}-1=1.12132^{+} \quad(n \in N) .
$$

Then they gave a new strengthened Hilbert's inequality which is the same as (1.6) with $p=2$. Since $\theta$ in (1.7) is not the best possible, Gau [5] obtained the best possible value of $\theta=\pi-\sum_{k=1}^{\infty} \frac{1}{(1+k)}\left(\frac{1}{\sqrt{k}}\right)=1.2811^{+}$. Subsequently, Gau [6] considered the general case and proved a new inequality for the weight coefficient $\omega(q, n)$ as

$$
\omega(q, n)<\frac{\pi}{\sin (\pi / p)}-\frac{\theta_{p}}{n^{1 / p}} \quad\left(q>1, \frac{1}{p}+\frac{1}{q}=1, n \in N\right),
$$

where $\theta_{p}=(p-1)$. Recently, Gau [7] replaced $(p-1)$ by $\theta_{p}=\theta_{p}(n)>0$ in (1.8). But the problem is that $\theta_{p}(n)$ depends on both $p$ and $q$. Simultaneously, Yang [8] found that $\theta_{p}=\theta=0.341295^{+}$, but the constant $\theta_{p}=\theta$ is not the best possible value. Finally, Yang and Gau [9] found the best possible value for $\theta_{p}=\theta=1-C=0.42278433^{+}$, where $C$ is a Euler constant. They also proved the following new Hardy-Hilbert's inequality

$$
\sum_{m=1}^{\infty} \sum_{n=1}^{\infty} \frac{a_{m} b_{n}}{m+n}<\left\{\sum_{n=1}^{\infty}\left[\frac{\pi}{\sin (\pi / p)}-\frac{1-C}{n^{1 / p}}\right] a_{n}^{p}\right\}^{1 / p}\left\{\sum_{n=1}^{\infty}\left[\frac{\pi}{\sin (\pi / p)}-\frac{1-C}{n^{1 / q}}\right] b_{n}^{q}\right\}^{1 / q}
$$

It is important to point out that (1.5) and (1.8) are different, and the constant $\eta_{p}$ in (1.5) depends on $p$.

The main objective of this paper is to prove an improved version of (1.5) as

$$
\omega(q, n)<\frac{\pi}{\sin (\pi / p)}-\frac{1}{2 n^{1 / p}+n^{-1 / q}} \quad\left(q>1, \frac{1}{p}+\frac{1}{q}=1, n \in N\right),
$$

and then prove a strengthened version of Hardy-Hilbert's inequality as follows:

$$
\sum_{m=1}^{\infty} \sum_{n=1}^{\infty} \frac{a_{m} b_{n}}{m+n}<\left\{\sum_{n=1}^{\infty}\left[\frac{\pi}{\sin (\pi / p)}-\frac{1}{2 n^{1 / p}+n^{-1 / q}}\right] a_{n}^{p}\right\}^{1 / p}\left\{\sum_{n=1}^{\infty}\left[\frac{\pi}{\sin (\pi / p)}-\frac{1}{2 n^{1 / q}+n^{-1 / p}}\right] b_{n}^{q}\right\}^{1 / q} .
$$

For this, we need the following inequality (see Yang [8] Lemma 1): If

$$
f(x)>0, f^{(2 r-1)}(x)<0, f^{(2 r)}(x) \geq 0, x \in[1, \infty)(r=1,2), f^{(r)}(\infty)=0(r=0,1,2,3,4),
$$

and $\int_{1}^{\infty} f(x) d x<\infty$, then

$$
\sum_{m=1}^{\infty} f(m) \leq \int_{1}^{\infty} f(x) d x+\frac{1}{2} f(1)-\frac{1}{12} f^{\prime}(1)
$$




\section{SOME LEMMAS}

LEMMA 2.1. If $q>1, p^{-1}+q^{-1}=1, n \in N$, then

$$
\omega(q, n)<\frac{\pi}{\sin (\pi / p)}-\frac{1}{n^{1 / p}}\left[f_{n}(p)+g_{n}(p)\right],
$$

where $\omega(q, n)$ is defined by $(1.5)$, and

$$
\begin{aligned}
& f_{n}(p):=p+\frac{1}{12 p}+\frac{1}{(1+p) n}+\frac{1}{12 p n^{2}}+\frac{1}{3(1+3 p) n^{3}} \\
& g_{n}(p):=\frac{-1}{12 p n}-\frac{1}{2(1+2 p) n^{2}}-\frac{7}{12}-\frac{1}{2 n}+\frac{1}{12 n^{2}}-\frac{7}{12 n^{3}} .
\end{aligned}
$$

PROOF. Let

$$
f(x)=\frac{1}{(x+n) x^{1 / q}}, \quad x \in[1, \infty)(q>1, n \in N) .
$$

By (1.12), we obtain that

$$
\sum_{m=1}^{\infty} \frac{1}{(m+n) m^{1 / q}} \leq \int_{1}^{\infty} \frac{1}{(x+n) x^{1 / q}} d x+\left(\frac{7}{12}-\frac{1}{12 p}\right) \frac{1}{1+n}+\frac{1}{12(1+n)^{2}} .
$$

Since

$$
\begin{aligned}
\int_{0}^{1 / n} \frac{1}{(1+y) y^{1 / q}} d y= & \int_{0}^{1 / n} \sum_{\nu=0}^{\infty}(-1)^{\nu} y^{\nu-1 / q} d y \\
= & \sum_{\nu=0}^{\infty}(-1)^{\nu} \int_{0}^{1 / n} y^{\nu-1 / q} d y=\frac{p}{n^{1 / p}} \sum_{\nu=0}^{\infty} \frac{(-1)^{\nu}}{(1+\nu p) n^{\nu}} \\
& >\frac{p}{n^{1 / p}} \sum_{\nu=0}^{3} \frac{(-1)^{\nu}}{(1+\nu p) n^{\nu}}=\frac{1}{n^{1 / p}}\left[p+\sum_{\nu=1}^{3} \frac{(-1)^{\nu}}{\nu n^{\nu}}-\sum_{\nu=1}^{3} \frac{(-1)^{\nu}}{\nu(1+\nu p) n^{\nu}}\right] .
\end{aligned}
$$

Putting $x=n y$, we find that

$$
\begin{aligned}
\int_{1}^{\infty} \frac{1}{(x+n) x^{1 / q}} d x & =\frac{1}{n^{1 / q}} \int_{1 / n}^{\infty} \frac{1}{(1+y) y^{1 / q}} d y \\
& =\frac{1}{n^{1 / q}}\left[\int_{0}^{\infty} \frac{1}{(1+y) y^{1 / q}} d y-\int_{0}^{1 / n} \frac{1}{(1+y) y^{1 / q}} d y\right] \\
& =\frac{1}{n^{1 / q}}\left[\frac{\pi}{\sin (\pi / p)}-\frac{p}{n^{1 / p}} \sum_{\nu=0}^{\infty} \frac{(-1)^{\nu}}{(1+\nu p) n^{\nu}}\right]^{\prime} \\
& <\frac{1}{n^{1 / q}} \frac{\pi}{\sin (\pi / p)}-\frac{1}{n}\left[p+\sum_{\nu=1}^{3} \frac{(-1)^{\nu}}{\nu n^{\nu}}-\sum_{\nu=1}^{3} \frac{(-1)^{\nu}}{\nu(1+\nu p) n^{\nu}}\right]
\end{aligned}
$$

we then find that

and

$$
\frac{1}{1+n}=\frac{1}{n}\left(1+\frac{1}{n}\right)^{-1}<\frac{1}{n}\left(1-\frac{1}{n}+\frac{1}{n^{2}}\right)
$$

$$
\frac{1}{(1+n)^{2}}=\frac{1}{n^{2}}\left(1+\frac{1}{n}\right)^{-2}<\frac{1}{n^{2}}\left(1-\frac{2}{n}+\frac{3}{n^{2}}\right)
$$

Substituting the above results in (2.2), by (1.5), we have (2.1). This proves the lemma. 
LEMMA 2.2. If $p>1, n \in N$, then

$$
f_{n}(p)+g_{n}(p)>\frac{1}{2}-\frac{1}{12 n}-\frac{1}{2 n^{3}} .
$$

PROOF. Since

and

$$
\begin{aligned}
f_{n}^{\prime}(p) & =1-\frac{1+n^{2}}{12 n^{2} p^{2}}-\frac{1}{(1+p)^{2} n}-\frac{1}{(1+3 p)^{2} n^{3}} \\
& >1-\frac{1+n^{2}}{12 n^{2}}-\frac{1}{(1+1)^{2} n}-\frac{1}{(1+3)^{2} n^{3}} \\
& =\frac{11}{12}-\frac{1}{12 n^{2}}-\frac{1}{4 n}-\frac{1}{16 n^{3}}>0,
\end{aligned}
$$

$$
g_{n}^{\prime}(p)=\frac{1}{12 p^{2} n}+\frac{1}{(1+2 p)^{2} n^{2}}>0,
$$

then $f_{n}(p)+g_{n}(p)$ is strictly increasing for $p \in(1, \infty)$, and

$$
f_{n}(p)+g_{n}(p)>\lim _{p \rightarrow 1}\left(f_{n}(p)+g_{n}(p)\right)=\frac{1}{2}-\frac{1}{12 n}-\frac{1}{2 n^{3}} .
$$

Thus the lemma is proved.

LEMMA 2.3. If $q>1, p^{-1}+q^{-1}=1, n \in N$, then inequality (1.10) is valid. So is the following inequality:

$$
\omega(p, n)<\frac{\pi}{\sin (\pi / p)}-\frac{1}{2 n^{1 / q}+n^{-1 / p}} .
$$

PROOF. Since for $n \geq 3$,

$$
\left(\frac{1}{2}-\frac{1}{12 n}-\frac{1}{2 n^{3}}\right)\left(1+\frac{1}{2 n}\right)=\frac{1}{2}+\frac{1}{n}\left(\frac{1}{6}-\frac{1}{24 n}-\frac{1}{2 n^{2}}-\frac{1}{4 n^{3}}\right)>\frac{1}{2},
$$

then

$$
\frac{1}{2}-\frac{1}{12 n}-\frac{1}{2 n^{3}}>\frac{1}{2+n^{-1}} \quad(n \geq 3)
$$

By (2.1) and (2.3), we have

$$
\begin{aligned}
\omega(q, n) & <\frac{\pi}{\sin (\pi / p)}-\frac{1}{n^{1 / p}}\left(\frac{1}{2}-\frac{1}{12 n}-\frac{1}{2 n^{3}}\right) . \\
& <\frac{\pi}{\sin (\pi / p)}-\frac{1}{2 n^{1 / p}+n^{-1 / q}} \quad(n \geq 3) .
\end{aligned}
$$

Taking $\theta_{p}=1-C$, by (1.8) (see Yang and Gau [9]), we find that

$$
\omega(q, 1)<\frac{\pi}{\sin (\pi / p)}-\frac{1-C}{1}<\frac{\pi}{\sin (\pi / p)}-\frac{1}{2 \times 1+1} .
$$

Since $C<3 / 5=0.6$, then we have

and

$$
\frac{1}{2 \times 2^{1 / p}+2^{-1 / q}}<\frac{1-C}{2^{1 / p}}
$$

$$
\omega(q, 2)<\frac{\pi}{\sin (\pi / p)}-\frac{1-C}{2^{1 / p}}<\frac{\pi}{\sin (\pi / p)}-\frac{1}{2 \times 2^{1 / p}+2^{-1 / q}} .
$$

It follows that for $n=1,2,(1.10)$ also holds. Then (1.10) is valid for any $n \in N$. Interchanging $p, q$ in (1.10), since $\frac{\pi}{\sin (\pi / p)}=\frac{\pi}{\sin (\pi / q)}$, we have (2.4). The lemma is proved. 
ON NEW STRENGTHENED HARDY-HILBERTS INEQUALITY

407

3. MAIN RESULTS

THEOREM 3.1. If $p>1, p^{-1}+q^{-1}=1, a_{n} \geq 0, b_{n} \geq 0$, and $0<\sum_{n=1}^{\infty} a_{n}^{p}<\infty$, $0<\sum_{n=1}^{\infty} b_{n}^{q}<\infty$, then inequality (1.11) is valid. We also have

$$
\sum_{m=1}^{\infty}\left(\sum_{n=1}^{\infty} \frac{a_{n}}{m+n}\right)^{p}<\left[\frac{\pi}{\sin (\pi / p)}\right]^{p-1} \sum_{n=1}^{\infty}\left[\frac{\pi}{\sin (\pi / p)}-\frac{1}{2 n^{1 / p}+n^{-1 / q}}\right] a_{n}^{p} .
$$

When $p=q=2$, this inequality reduces to the form

$$
\sum_{m=1}^{\infty}\left(\sum_{n=1}^{\infty} \frac{a_{n}}{m+n}\right)^{2}<\pi \sum_{n=1}^{\infty}\left[\pi-\frac{1}{2 \sqrt{n}+\sqrt{n^{-1}}}\right] a_{n}^{2} .
$$

PROOF. By Holder's inequality, we have

$$
\begin{aligned}
\sum_{m=1}^{\infty} \sum_{n=1}^{\infty} \frac{a_{m} b_{n}}{m+n} & =\sum_{m=1}^{\infty} \sum_{n=1}^{\infty}\left[\frac{1}{(m+n)^{1 / p}}\left(\frac{m}{n}\right)^{1 / p q} a_{m}\right]\left[\frac{1}{(m+n)^{1 / q}}\left(\frac{n}{m}\right)^{1 / p q} b_{n}\right] \\
& \leq\left\{\sum_{m=1}^{\infty} \sum_{n=1}^{\infty} \frac{1}{m+n}\left(\frac{m}{n}\right)^{1 / q} a_{m}^{p}\right\}^{1 / p}\left\{\sum_{m=1}^{\infty} \sum_{n=1}^{\infty} \frac{1}{m+n}\left(\frac{n}{m}\right)^{1 / p} b_{n}^{q}\right\}^{1 / q} \\
& =\left\{\sum_{n=1}^{\infty}\left[\sum_{m=1}^{\infty} \frac{1}{m+n}\left(\frac{n}{m}\right)^{1 / q}\right] a_{n}^{p}\right\}^{1 / p}\left\{\sum_{n=1}^{\infty}\left[\sum_{m=1}^{\infty} \frac{1}{m+n}\left(\frac{n}{m}\right)^{1 / p}\right]_{n}^{q q}\right\}^{1 / q} \\
& =\left\{\sum_{n=1}^{\infty} \omega(q, n) a_{n}^{p}\right\}^{1 / p}\left\{\sum_{n=1}^{\infty} \omega(p, n) b_{n}^{q}\right\}^{1 / q} .
\end{aligned}
$$

Hence, by (1.10) and (2.4), inequality (1.11) holds.

Since by $(2.4), \omega(p, n)<\frac{\pi}{\sin (\pi / p)}$, then by Holder's inequality, we obtain

$$
\begin{aligned}
\sum_{n=1}^{\infty} \frac{a_{n}}{m+n} & =\sum_{n=1}^{\infty}\left[\frac{a_{n}}{(m+n)^{1 / p}}\left(\frac{n}{m}\right)^{1 / p q}\right]\left[\frac{1}{(m+n)^{1 / q}}\left(\frac{m}{n}\right)^{1 / p q}\right] \\
& \leq\left\{\sum_{n=1}^{\infty}\left[\frac{1}{m+n}\left(\frac{n}{m}\right)^{1 / q}\right] a_{n}^{p}\right\}^{1 / p}\left\{\sum_{n=1}^{\infty} \frac{1}{m+n}\left(\frac{m}{n}\right)^{1 / p}\right\}^{1 / q} \\
& =\left\{\sum_{n=1}^{\infty}\left[\frac{1}{m+n}\left(\frac{n}{m}\right)^{1 / q}\right] a_{n}^{p}\right\}^{1 / p}\{\omega(p, n)\}^{1 / q} . \\
& <\left\{\sum_{n=1}^{\infty}\left[\frac{1}{m+n}\left(\frac{n}{m}\right)^{1 / q}\right] a_{n}^{p}\right\}^{1 / p}\left\{\frac{\pi}{\sin (\pi / p)}\right\}^{1 / q} .
\end{aligned}
$$

By (1.10), we find

$$
\begin{aligned}
\sum_{m=1}^{\infty}\left(\sum_{n=1}^{\infty} \frac{a_{n}}{m+n}\right)^{p} & <\left[\frac{\pi}{\sin (\pi / p)}\right]^{p / q} \sum_{m=1}^{\infty} \sum_{n=1}^{\infty} \frac{1}{m+n}\left(\frac{n}{m}\right)^{1 / q} a_{n}^{p} \\
& =\left[\frac{\pi}{\sin (\pi / p)}\right]^{p-1} \sum_{n=1}^{\infty}\left[\sum_{m=1}^{\infty} \frac{1}{m+n}\left(\frac{n}{m}\right)^{1 / q}\right] a_{n}^{p} \\
& =\left[\frac{\pi}{\sin (\pi / p)}\right]^{p-1} \sum_{n=1}^{\infty} \omega(q, n) a_{n}^{p} \\
& <\left[\frac{\pi}{\sin (\pi / p)}\right]^{p-1} \sum_{n=1}^{\infty}\left[\frac{\pi}{\sin (\pi / p)}-\frac{1}{2 n^{1 / p}+n^{-1 / q}}\right] a_{n}^{p} .
\end{aligned}
$$

This proves result (3.1). Thus the proof of Theorem 3.1 is complete. 


\section{CONCLUDING REMARKS}

(a) Inequality (1.11) is a definite improvement over (1.6).

(b) Since, for $n \geq 3, C>\left(\frac{n+1}{2 n+1}\right)$, then

$$
\frac{\pi}{\sin (\pi / p)}-\frac{1}{2 n^{1 / p}+n^{-1 / q}}<\frac{\pi}{\sin (\pi / p)}-\frac{(1-C)}{n^{1 / p}}, \quad(n \geq 3) .
$$

In view of (2.5), (2.6) and (3.3), it follows that (1.9) and (1.11) represent two distinct versions of strengthened inequalities. But they are not comparable.

(c) Inequality (3.1) reduces to

$$
\sum_{m=1}^{\infty}\left(\sum_{n=1}^{\infty} \frac{a_{n}}{m+n}\right)^{p}<\left[\frac{\pi}{\sin (\pi / p)}\right]^{p} \sum_{n=1}^{\infty} a_{n}^{p},
$$

This is an equivalent form of Hardy-Hilbert's inequality (1.4) (see Hardy et al. [11], Chapter 9).

\section{REFERENCES}

[1] MIKHILIN, S.G., Constants in Some Inequalities of Analysis, John Wiley \& Sons, New York, 1986.

[2] XU, L.C. and GAU, Y.K., Note on Hardy-Riesz's extension of Hilbert's inequality, Chinese Quarterly Journal of Mathematics, 6, 1 (1991), 75-77.

[3] HSU, L.C. and WANG, Y.J., A refinement of Hilbert's double series theorem, J. Math. Res. Exp. 11, 1 (1991), 143-144.

[4] ZHAO, D.J., On a refinement of Hilbert's double series theorem, Math. Practice and Theory, 1 (1993), 85-90.

[5] GAU, M.Z., A note on Hilbert double series theorem, Hunan Annals of Mathematics, 12, 1-2 (1992), 142-147.

[6] GAU, M.Z., An improvement of Hardy-Riesz's extension of the Hilbert inequality, J. Math. Res. Exp. 14, 2 (1994), 255-259.

[7] GAU, M.Z., A note on the Hardy-Hilbert inequality, J. Math. Ana. Appl. 204 (1996), 346-351.

[8] YANG, B.C., A refinement on the general Hilbert's double series theorem, J. Math. Study, 29, 2 (1996), 64-70.

[9] YANG, B.C. and GAU, M.Z., On a best value of Hardy-Hilbert's inequality, Advances in Math., 26, 2 (1997), 159-164.

[10] YANG, B.C. and DEBNATH, L., Some inequalities involving the constant $e$, and an application to Carleman's inequality, J. Math. Anal. and Appl., to appear (1997).

[11] HARDY, G.H., LITTLEWOOD, J.E. and POLYA, G., Inequalities, Cambridge University Press, Cambridge, 1952. 


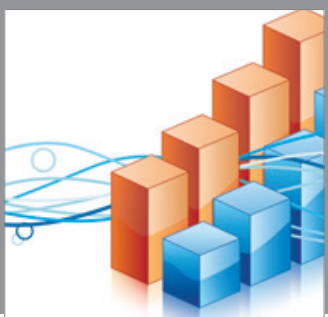

Advances in

Operations Research

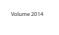

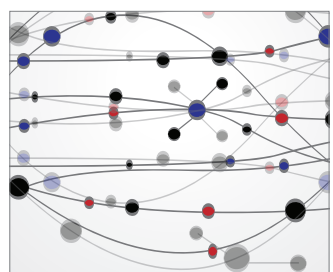

\section{The Scientific} World Journal
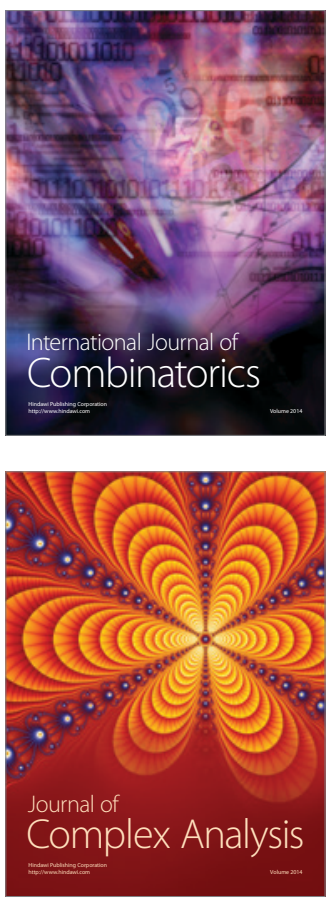

International Journal of

Mathematics and

Mathematical

Sciences
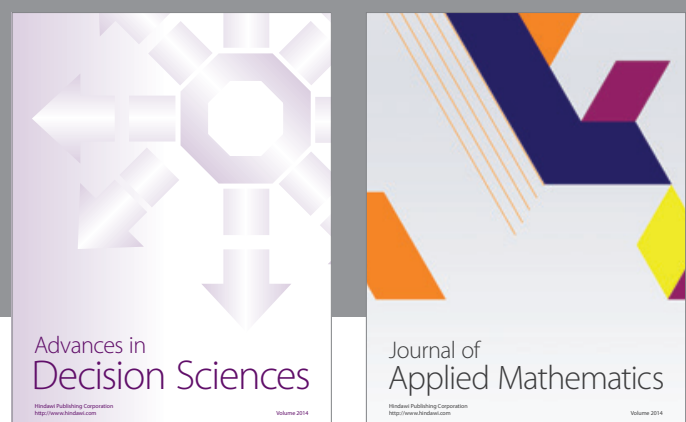

Journal of

Applied Mathematics
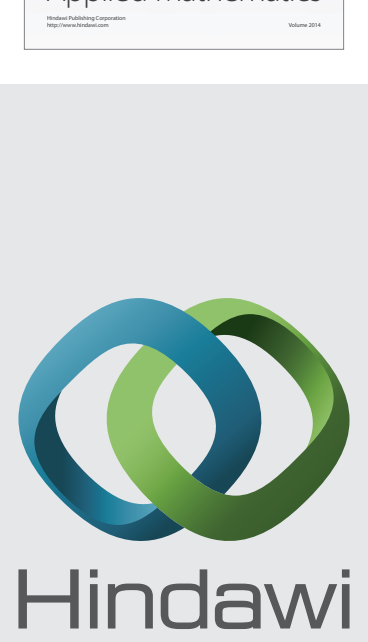

Submit your manuscripts at http://www.hindawi.com
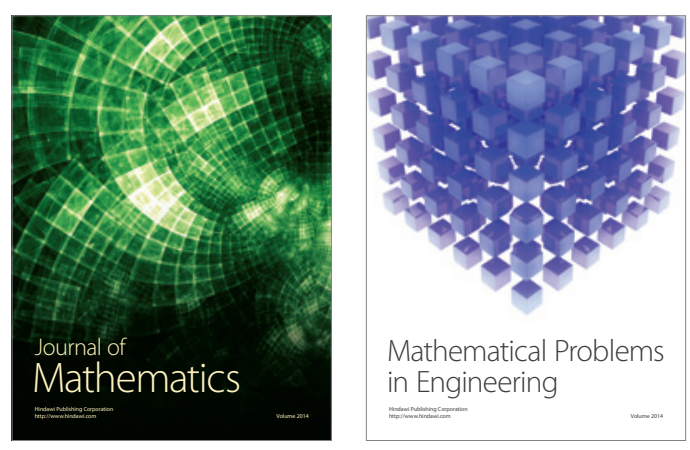

Mathematical Problems in Engineering
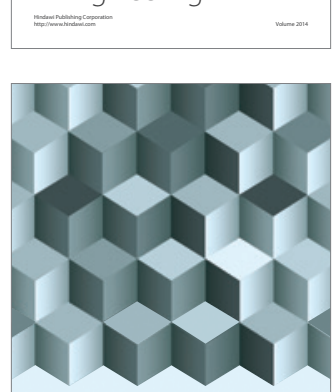

Journal of

Function Spaces
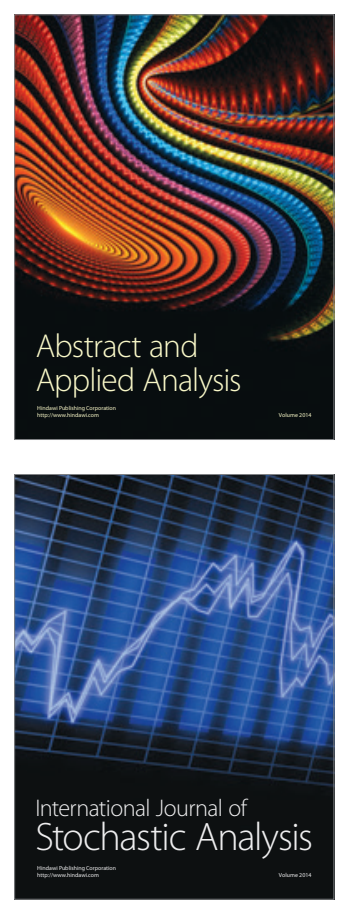

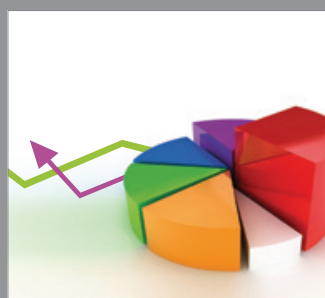

ournal of

Probability and Statistics

Promensencen
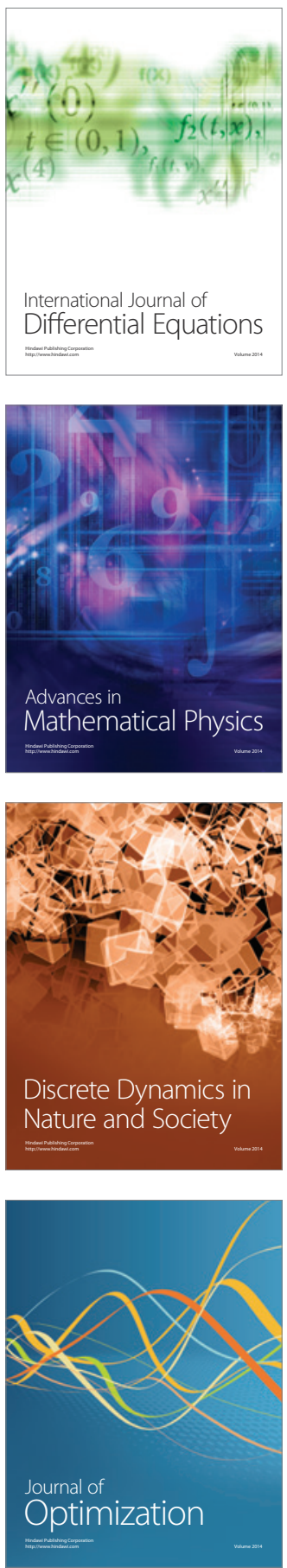